\title{
Efficacy of Constraint Induced Movement Therapy and Bilateral Arm Training to Improve Upper Limb Motor Functions in HemipareticSubjects - Comparative Study
}

\section{J. Blessy Jasmine, ${ }^{\text {* }}$ P.G. Mahesh Kumar, ${ }^{2}$ Jagatheesan Alagesan, ${ }^{3}$ Iyyappan Manickavasagam ${ }^{4}$}

1. Associate Professor, Meenakshi Faculty of Physiotherapy, Meenakshi Academy of Higher Education and Research, Chennai, India.

2. Assistant Professor, Meenakshi Faculty of Physiotherapy, Meenakshi Academy of Higher Education and Research, Chennai, India.

3. Assistant Professor in Physiotherapy, College of Health Sciences, Gulf Medical University, Ajman, United Arab Emirates.

4. Senior Physiotherapist, Dubai Physiotherapy \& Rehabilitation Centre, Al Safa, Dubai * - Corresponding author

\section{Abstract}

Stroke is a sudden loss of brain function due to the interruption of its blood flow or the rupture of the blood vessels in the brain. It involves disruption in the motor, somatosensory, visual, speech, perceptual, cognitive, bowel and bladder dysfunctions. It leads to muscle tone alterations, muscle weakness and abnormal movements which consequently limit the ability to perform functional activities including self-care. This study aims to compare the effectiveness of Constraint Induced Movement Therapy with Bilateral Arm Training to improve upper limb motor functions in hemiparetic subjects. The CIMT group concentrated on repetitive task practice and adaptive task practice. The BAT group concentrated on the simultaneous movements of both the affected and unaffected upper limb in functional tasks in symmetric or alternating patterns. Thirty stroke survivors of both genders were included in this quasi experimental study with the age ranging 30 to 50 years who were medically stable, able to communicate properly, and diagnosed with ischemic stroke of more than 6 months duration. The outcome measures are Upper extremity Motor Activity Log and Fugyl-Meyer Assessment. The results from this study concluded the effectiveness of six weeks of constraint induced movement therapy could significantly improve the upper limb motor functions and functional activities in hemi paretic stroke patients. Hence the Constraint Induced Movement Therapy program had better therapeutic effect and significantly improves the movement capabilities of hemi paretic patients.

Key Words:Stroke, Constraint Induced Movement Therapy, Bilateral Arm Training, Motor Activity Log, Fugyl-Meyer Scale

\section{Introduction}

Stroke is a sudden loss of brain function due to the interruption of its blood flow or the rupture of the blood vessels in the brain. It involves disruption in the motor, somatosensory, visual, speech, 
perceptual, cognitive, bowel and bladder dysfunctions. It leads to muscle tone alterations, muscle weakness and abnormal movements which consequently limit the ability to perform functional activities including self-care. ${ }^{1}$ Stroke is one of the major cause for death and the most important single cause of severe disability in the community making it an important concern for rehabilitation team. ${ }^{2}$

People with stroke show an impaired upper extremity function due to muscle weakness and unable to move isolated segments of the limb without producing movements in the rest of the limb. Upper limb weakness inhemi paresis is a major factor restricting functional recovery in more than 85 percent of stroke patients. Reduced upper limb function has more impact on ability to perform activities of daily living, which is likely to reduce independence and increase burden of care. The unsuccessful use of the affected Upper limb in stroke patients may cause 'learned nonuse phenomenon' in which the patient habitually relay on their unaffected upper limb to accomplish activities of daily living. Improving the paretic upper extremity functional activities is a major objective in the rehabilitation program for stroke subjects. ${ }^{3,4}$

Constraint Induced Movement Therapy is a multifaceted neuro rehabilitation technique that aims to improve motor function and increase the use of the hemiparetic upper limb for daily activities. ${ }^{5,6,7}$ The therapy derives from the concept of learned nonuse of a limb resulting from peripheral or central nervous system injury which was described by Taub. ${ }^{3,4}$ Overcoming this learned behaviour by shaping its movement through interactive attempts to accomplish tasks and forcing its use through restraint of the contra lateral limb. ${ }^{43}$ This may be achieved by intense repetitive task oriented training of affected upper limb and immobilization of the unaffected upper limb.

An alternative neuro rehabilitation program that is gaining increasing attention is Bilateral Arm Training which employs the repetitive practice of symmetrical bilateral movement to improve motor performance of the affected upper limb. The bilateral arm training emphasis on use of simultaneous movements in symmetric or alternating patterns of affected and unaffected upper limb for functional tasks. These functional tasks also emphasize the upper limb movements involved in daily activities, but focuses on both upper limbs moving synchronously. Bilateral arm training helps to facilitate unilateral skill recovery through reduced intra cortical inhibition and increased intra cortical facilitation in both hemispheres. ${ }^{12}$ Thisstudy aims to compare the effectiveness of Constraint Induced Movement Therapywith Bilateral Arm Training to improve upper limb motor functions in hemipareticsubjects.

\section{Method}

Thirty stroke survivors of both genders were included in this quasi experimental study with the age ranging 30 to 50 years who were medically stable, able to communicate properly, and diagnosed with ischemic stroke of more than 6 months duration. The other criteria to include in this study are Brunnstorm voluntary control grading stage above three for upper limb spasticity of Modified Ashworth Scale score less than two. Subjects with severe other impairments prior to stroke, causing persistent disability and patients with cognitive and perceptual impairments were excluded. The study was conducted in a physiotherapy teaching institute and was cleared by institutional review board; all participants signed an informed consent prior to participation. The subjects were randomly divided into two groups with 15 in each the Constraint Induced Movement Therapy (CIMT) Group and Bilateral Arm Training (BAT) Group. The outcome measures used are Motor Activity Log and Fugyl-Meyer Assessment Scale before and after the intervention as specified for the allotted group. The intervention for both groups was given for two hours per day, five days per week for six weeks.

Upper extremity Motor Activity Log is a structured interview intended to examine How Much (Amount Scale) and How Well (How Well Scale) the subject uses their more affected arm outside the 
laboratory setting. Participants are asked standardized questions about the amount of use of their more affected arm and the quality of their movement during the functional activities indicated. ${ }^{13,14}$

The Fugyl-Meyer Assessment is a stroke specific, performance based impairment index. It is designed to assess motor functioning, balance, sensation, joint range of motion and joint pain in patients with post stroke hemiparesis.Scale items are scored on the basis of ability to complete the item using a 3 point ordinal scale where $0=$ cannot perform, $1=$ performs partially and $2=$ performs fully, with the maximum score of 226 points. The scoring for the upper extremity is taken in this study. ${ }^{15,16}$

The CIMT group concentrated on repetitive task practice and adaptive task practice. Repetitive task practice refers to continuous efforts to execute movements that usually are repeated, for example, eating, grooming, or brushing teeth. Adaptive task practice is characterized by repetitions of a defined movement, such as picking up blocks and moving them toward a pail, in a series of trials. Each trial has a defined duration and increased in successful number of repetitions or to reduce the time to complete the task demands successfully with one effort. ${ }^{8,17}$

The BAT group concentrated on the simultaneous movements of both the affected and unaffected upper limb in functional tasks in symmetric or alternating patterns such as lifting two cups, picking up two pegs, reaching forward or upward to move blocks, grasping and releasing two towels. ${ }^{12,17}$

\section{Data Analysis}

There were 30 subjects with the mean age of 45.7 years. 13 subjects were right side affected (6 in CIMT and 7 in BAT) and 19 were males (10 in CIMT and 9 in BAT). The data analysis was computed with SPSS-17. Wilcoxon signed ranks test was used to test the significant difference within the groups and Mann Whitney U test was used to test the significant difference between groups. The level of significance was fixed at 0.05 for all analysis. At baseline both the groups were homogenous in all outcome measures with $\mathrm{p}$ more than 0.05 .

Table-1: Within group analysis for both groups for both outcome measures

\begin{tabular}{|c|c|c|c|c|c|}
\hline Outcome & \multicolumn{2}{|c|}{ Intervention } & Mean & SD & $\mathrm{p}$ \\
\hline \multirow{4}{*}{ FMA Scores } & \multirow[t]{2}{*}{ CIMT } & Before & 101.9 & 8.145 & \multirow[t]{2}{*}{$<0.001$} \\
\hline & & After & 121.4 & 5.179 & \\
\hline & \multirow[t]{2}{*}{ BAT } & Before & 104.4 & 10.41 & \multirow[t]{2}{*}{$<0.001$} \\
\hline & & After & 111.06 & 7.84 & \\
\hline \multirow{4}{*}{$\begin{array}{l}\text { MAL Scores } \\
\text { Amount Scale }\end{array}$} & \multirow[t]{2}{*}{ CIMT } & Before & 53.6 & 4.28 & \multirow[t]{2}{*}{$<0.001$} \\
\hline & & After & 64.6 & 5.65 & \\
\hline & \multirow[t]{2}{*}{ BAT } & Before & 51.06 & 1.830 & \multirow[t]{2}{*}{$<0.001$} \\
\hline & & After & 61.3 & 2.126 & \\
\hline \multirow{4}{*}{$\begin{array}{l}\text { MAL Scores } \\
\text { How Well Scale }\end{array}$} & \multirow[t]{2}{*}{ CIMT } & Before & 63.7 & 4.60 & \multirow[t]{2}{*}{$<0.001$} \\
\hline & & After & 72.4 & 5.27 & \\
\hline & \multirow[t]{2}{*}{ BAT } & Before & 62.6 & 2.89 & \multirow[t]{2}{*}{$<0.001$} \\
\hline & & After & 65.8 & 3.62 & \\
\hline
\end{tabular}

Table 1 show within group analyses before and after intervention for both groups in all outcome measures. All the outcome measures proved statistically significant improvement with $p$ value less than 0.05 before and after intervention in both groups. 
Table-2: Between group analysis after intervention for both groups

\begin{tabular}{|l|l|l|l|l|}
\hline Outcome & Intervention & Mean & SD & \multirow{2}{*}{ p } \\
\hline FMA Scores & CIMT & 121.4 & 5.179 & \multirow{2}{*}{$<0.001$} \\
\cline { 2 - 4 } & BAT & 111.06 & 7.84 & \multirow{2}{*}{.021} \\
\hline $\begin{array}{l}\text { MAL Scores } \\
\text { Amount Scale }\end{array}$ & CIMT & 64.6 & 5.65 & \\
\cline { 2 - 4 } $\begin{array}{l}\text { MAL Scores } \\
\text { How Well Scale }\end{array}$ & BAT & 61.3 & 2.126 & \multirow{2}{*}{.011} \\
\cline { 2 - 4 } & CIMT & 72.4 & 5.27 & \\
\cline { 2 - 4 } & BAT & 65.8 & 3.62 & \\
\hline
\end{tabular}

Table 2 shows the analyses between groups after intervention. There is no statistically significant difference among the groups after intervention but the CIMT group showed more mean scores in all the outcome measures indicating better improvement in CIMT group than the BAL group.

\section{Discussion}

Interpreting the results of the present study shows that both Constraint Induced Movement Therapy and Bilateral Arm Training are effective in improving the Upper Limb motor functions in hemiparetic stroke patients. The difference between the two groups in favour of the motor score, sensation and joint pain with Constraint Induced Movement Therapyshowed higher improvement, however indicates that different physiotherapy approaches have an impact on the results of the early rehabilitation ofhemiparetic stroke patients.

Both the groups have shown improvement in FMA scale and in MAL scale after the treatment. Rationale behind improvement in functional activities might be due to decrease in joint pain, increased range of motion and improved grasping power which lessened suffering in daily activities, and difficulty in moving the affected extremity. Both groups had improved their joint range of motion and reduction in joint pain, whereas CIMT group improved slightly more than BAT group in terms of functional activities.

CIMT group were given constraint induced movement therapy which improve motor activities through Intense repetitive task oriented training of impaired affected upper limb and Immobilization of the unimpaired upper limb and Shaping which includes both repetitive task practice and adaptive task practice. Repetitive task practice refers to continuous efforts to execute movements that usually are repeated, for example, eating, grooming, or brushing teeth. Adaptive task practice is a form of operant or instrumental conditioning characterized by repetitions of a defined movement, such as picking up blocks and moving them toward a pail, in a series of trials. ${ }^{8-10}$

Although the constraint induced movement therapy has been shown to modify brain activity, especially in the affected motor and premotor cortexes, and that interconnections from undamaged hemispheric structures, through which constraint induced movement therapy can induce neuroplasticity. ${ }^{18}$ Asaresultthattheuseofpareticlimbincreasescorticalrepresentation for movements directed away from a hand and wrist synergy and that metabolic activity changes can be observed in the primary and pre motor cortex, cerebellum and other structures linked to motor outflow from either the ipsilesional or thecontralesional brain.

Several studies have demonstrated about Constraint Induced Movement Therapy training in which the non-paretic arm is restrained to enforce to task oriented exercises with the paretic limb, reorganization in the cerebellum is reported to occur after Constraint Induced Movement Therapy. ${ }^{9,11}$ The person with motor impairments following a neural lesion needs to learn again how to control 
segmental movement, so that the spatial configuration and temporal sequencing of body movements brings about an effective motion, thereby achieving the individual's goal with energy expenditure.

Also this study was supported by Levy and colleagues who were first to report on changes in brain activation in two patients with chronic stroke who received Constraint Induced Movement Therapy. Using functional MRI they showed that gains in motor function after Constraint Induced Movement Therapy were accompanied by increased activation in the contralesional hemisphere and peri infarct zone in one of the patients, and in the ipsilesional hemisphere in the other patients. ${ }^{18}$ This study was supported by authors Dong and colleagues showed that changes in brain activation during the course of the 2 week therapy varied over time and among individual stroke patients.

A systematic review of randomized controlled trials of CIMT in stroke concluded that Constraint Induced Movement Therapy can improve upper limb function following stroke for some patients when compared to alternative or no treatment (Hakkennes S, Keating J, 2007). ${ }^{19}$ Previous results from the excite trial have shown that Constraint Induced Movement Therapy can produce statistically and clinically meaningful improvements in hand function after the intervention and 1 year later compared with those in participants who receive usual and customary care (Wolf SL et al 2006). ${ }^{4}$

The affected arm may act as a dead weight, dragging on and changing trunk alignment, therebymaking it difficult to balance on affected leg. Increased functional use of the affected hand after CIMT may have relived the negative impact on trunk alignment and balance control and thus resulted in improved locomotion.BAT group was given bilateral arm training, which employs the repetitive practice of symmetrical bilateral movement to improve motor performance of the affected upper limb. The BAT group concentrated on the simultaneous movements of both the affected and unaffected Upper Limb in functional tasks in symmetric or alternating patterns. These functional tasks also emphasized upper limb movements involved in daily activities, but focused on both ULs moving synchronously, such as lifting 2 cups, picking up 2 pegs, reaching forward or upward to move blocks, grasping and releasing 2 towels. ${ }^{12,20,21}$

Some studies demonstrated that Bilateral Arm Training induced changes in movement related cortical activation patterns in chronic stroke survivors, suggesting cortical reorganization. Increased recruitment was observed in sensorimotor area of the contralesional hemisphere and in the ipsilesional cerebellum. ${ }^{7}$ In patients with such changes arm function improved. Several studies included both unilateral and bilateral training or coupled bilateral training with other training approaches but there is no systematic attempt to determine whether this augments the response benefit for all levels of patients( McCombe Waller and Jill Whitall 2008). ${ }^{12}$

Bilateral Arm Training facilitatesreduced intra cortical inhibition and increased intra cortical facilitation in both hemispheres, while unilateral training [dominant/ non dominant] produced increased intra cortical facilitation and reduced intra cortical inhibition in the contra lesional hemisphere. ${ }^{12}$ Practice of training the unilateral non paretic arm alone for stroke patients may reinforce inhibitory process in the lesioned hemisphere that could impede recovery of paretic arm.

The present study demonstrated important findings that, 6 weeks structured exercise training program in more than 6 months of post stroke hemiparetic patients could significantlyimprove their functional activities. All subjects performed voluntary hand movements on their non-paretic side during the therapy sessions. In this study we used different tasks to improve their functional activities and auditory feedback to enhance motor learning. There is a considerable improvement in both the groups and the improvement is more in the CIMT group. 


\section{Conclusion}

The results from this study concluded the effectiveness of six weeks of constraint induced movement therapy could significantly improve the upper limb motor functions and functional activities in hemi paretic stroke patients. Hence the Constraint Induced Movement Therapy program had better therapeutic effect and significantly improves the movement capabilities of hemi paretic patients.

\section{References}

1. SankaraKumaranPandian, Jagatheesan Alagesan. Effect of Mental Imagery Training for subjects with Stroke. International Journal of Scientific Research. 2013;2(8): 392-393.

2. Jagatheesan Alagesan, Surbala L. Devi, VaiyapuriAnandh. Comparative Study on the Strength of Ipsilateral Upper Extremity of Stroke Subjects and Normal Individuals. International Journal of Pharmaceutical Science and Health Care. 2011;1(3):22-32.

3. Wolf SL, Lecraw DE, Barton LA, Jann BB. Forced use of hemiplegic upper extremities to reverse the effect of learned nonuse among chronic stroke and head -injured patients. Exp Neurol. 1989;104:125-132.

4. Wolf SL,Winstein CJ, Miller JP,Taub E,Uswatte G, Morris, et al.Effect of constraintinduced movement therapy on upper extremity function 3 to 9 months after stroke:the excite randomized clinical trial. JAMA 2006;296:2095-2104.

5. Blanton S,Wilsey H,Wolf SL. Constraint induced movement therapy in stroke rehabilitation:perspectives on future clinical application.Neurorehabil 2008;23:15-28.

6. Boake C,Noser EA,Ro T,Baraniuks,Gaber M , Johnson R,et al. Constraint induced movement therapy during early stroke rehabilitation. Neurorehabil Neural repair. 2007;21:14-24

7. Taub E, Crago JE, Uswatte G. Constraint-induced movement therapy:a new approach to treatment in physical rehabilitation. Rehabil psycho. 1998;43:152-70.

8. Biitefisch C, Hummelsheim H,Denzler P, Mauritz KH.Repetitive training of isolated movement improves the outcome of motor rehabilitation of the centrally paretic hand. J NeurolSci 1995; 130:59-68.

9. C.G. Ostendorf, S.L. Wolf. Effect of forced use of the upper extremity of a hemiplegic patient on changes in function. A single case design. Physical Therapy1981, 1022-1028.

10. Taub E. Constraint induced movement therapy and massed practice [letter to the editor]. Stroke. 2000; 31:983.

11. Taub E,Wolf SL.Constraint induced movement techniques to facilitate upper extremity use in stroke patients . Top stroke rehabil 1997;3:38-61.

12. McCombe Waller S, Forrester LW, Villagra F. Intracorticalinhibition and facilitation with unilateral dominant, unilateral non dominant and bilateral movement tasks in left and right handed adults. JNeurol Sci. 2008;269:96-104.

13. A. Johnson, L. Judson, D. Morris, G. Uswatte and E. Taub. The validity and reliability of the 45- item upper extremity motor activity log, presented at the American Physical Therapy Association., combined sections meeting, Nashville, 2004.

14. Park SW,Wolf SL,Blanton S, Winstein C, Nichols Larsen DS. The EXCITE Trial:predicting clinically meaningfull motor activity log outcome.Neurorehabil Neural Repair 2008;22:486493.

15. Fugl- Meyer A, Jaasko L, Leyman I, Olsson S, SteglindS. The post stroke hemiplegic patient: a method for evaluation of physical performance. Scand J Rehabil Med. 1975;7: 13-31. 
16. Sanford J, Moreland J, Swanson LR, Stratford PW, Gowland C. Reliability of the fugl- Meyer assessment for testing motor performance in patients following stroke. Physther. 1993; 73: 447454.

17. Summers JJ, Kagerer FA, Garry MI, Hiraga CY, Loftus A. Bilateral and unilateral movement training on upper limb function in chronic stroke patients: a TMS study: J Neurol Sci. 2007:252:76-82.

18. Levy CE, Nichols DS, Schmalbrock PM, Keller P, Chakeres DW. Functional MRI evidence of cortical reorganization in upper-limb stroke hemiplegia treated with constraint induced movement therapy. Am J Phys Med Rehabil 2001; 80: 4-12.

19. Hayner K, Gibson G ,Gilses GM .Comparison of constraintinduced movement therapy and bilateral treatment of equal intensity in people with chronic upper extremity dysfunction after cerebrovascular accident.Am J OccupTher 2010;64:528-539.

20. Kopp B, Kunkel A,Muhlnickel W,Villringer K, Taub E, Flor H. Plasticity in the motor system related to therapy induced improvement of movement after stroke. Neuroreport 1999;10:80710.

21. Cauragh JH, Summers JJ. Neural plasticity and bilateral movements: a rehabilitation approach for chronic stroke. Progneurobiol. 2005;5:309-320. 